Article

\title{
Quantifying the Impacts of Environmental Factors on Vegetation Dynamics over Climatic and Management Gradients of Central Asia
}

\author{
Olena Dubovyk ${ }^{1, *}$, Tobias Landmann ${ }^{2}$, Andreas Dietz ${ }^{3}$ and Gunter Menz ${ }^{1,4}$ \\ 1 Center for Remote Sensing of Land Surfaces (ZFL), University of Bonn, Bonn 53113, Germany; \\ gmenz@uni-bonn.de \\ 2 Earth Observation Unit, International Center of Insect Physiology and Ecology (ICIPE), Duduville, \\ Kasarani Road, P.O. Box 30772, Nairobi 00100, Kenya; tlandmann@icipe.org \\ 3 German Remote Sensing Data Center (DFD), German Aerospace Center (DLR), Wessling 82234, Germany; \\ andreas.dietz@dlr.de \\ 4 Remote Sensing Research Group, Department of Geography, University of Bonn, Meckenheimer Allee 166, \\ Bonn 53115, Germany \\ * Correspondence: odubovyk@uni-bonn.de; Tel.: +49-228-73-7198
}

Academic Editors: Petri Pellikka, Lars Eklundh, Parth Sarathi Roy and Prasad S. Thenkabail Received: 17 May 2016; Accepted: 13 July 2016; Published: 15 July 2016

\begin{abstract}
Currently there is a lack of quantitative information regarding the driving factors of vegetation dynamics in post-Soviet Central Asia. Insufficient knowledge also exists concerning vegetation variability across sub-humid to arid climatic gradients as well as vegetation response to different land uses, from natural rangelands to intensively irrigated croplands. In this study, we analyzed the environmental drivers of vegetation dynamics in five Central Asian countries by coupling key vegetation parameter "overall greenness" derived from Moderate Resolution Imaging Spectroradiometer (MODIS) Normalized Difference Vegetation Index (NDVI time series data, with its possible factors across various management and climatic gradients. We developed nine generalized least-squares random effect (GLS-RE) models to analyze the relative impact of environmental factors on vegetation dynamics. The obtained results quantitatively indicated the extensive control of climatic factors on managed and unmanaged vegetation cover across Central Asia. The most diverse vegetation dynamics response to climatic variables was observed for "intensively managed irrigated croplands". Almost no differences in response to these variables were detected for managed non-irrigated vegetation and unmanaged (natural) vegetation across all countries. Natural vegetation and rainfed non-irrigated crop dynamics were principally associated with temperature and precipitation parameters. Variables related to temperature had the greatest relative effect on irrigated croplands and on vegetation cover within the mountainous zone. Further research should focus on incorporating the socio-economic factors discussed here in a similar analysis.
\end{abstract}

Keywords: land surface dynamics; time-series data; vegetation patterns; Moderate Resolution Imaging Spectroradiometer (MODIS); linear regression modeling

\section{Introduction}

Countries in post-Soviet Central Asia (CA) have undergone dramatic geopolitical, socio-economic, and environmental changes during the last decades. The collapse of the Soviet Union in 1991 resulted in extensive changes in land uses and land covers (LULC) due to ongoing economic and political transformations within the region [1]. Extensive irrigation systems established within CA have drastically altered the environment, particularly in the downstream areas of the region's principal rivers, the Amu Darya and the Syr Darya [2]. Ongoing socioeconomic processes (including migration) 
combined with cropland degradation have caused widespread abandonment of agricultural land $[3,4]$. Finally, considering the predicted increasing scarcity of water resources resulting from global climate change and population growth [5], understanding LULC change dynamics becomes critically important for this region.

Optical remote sensing provides the most cost-effective technique for monitoring and characterizing land cover dynamics over extensive areas. Traditional alternative methods which rely on in situ analyses are very resource demanding. Several systematic techniques have been developed to perform vegetation change detection analyses using remote sensing.

1. Algorithms are applied that measure either spectral differences among individual images or image-derived vegetation indices such as regression analysis and change vector analysis [6,7]. This group of algorithms measure gradual changes between two or more images.

2. Other algorithms are based on image classification through comparison of multi-temporal LULC maps to identify changes in mapped classes [8,9].

3. Vegetation phenology parameters such as growing season onset, peak, and end are first calculated and then their spatio-temporal dynamics are analyzed over the given observation period [10,11].

In relation to the temporal dimension of such analyses, they can be performed in bi-temporal (first and second groups), multi-temporal (all groups) or hyper-temporal manner (first and third groups).

Land cover dynamics function as an integrated indicator of vegetation responses to environmental factors such as rainfall, temperature, soil, and topography as well as factors related to human activities, which can be typically derived from LULC information (e.g., irrigated agriculture). Linking vegetation cover dynamics with climatic and anthropogenic factors can facilitate an improved understanding of vegetation cover changes as well as ecosystem feedbacks to natural stresses and human activities.

Recently, a number of studies have focused on analyses of LULC at local to regional scales in CA. The studies [12-15] all analyzed vegetation changes and their relationships typically to two factors: temperature and precipitation based on long-term advanced very high resolution radiometer (AVHRR) normalized difference vegetation index (NDVI) data. De Beurs et al. [16] assessed LULC changes in Kazakhstan using AVHRR-NDVI satellite time series. Land degradation and relations between degradation and possible triggers (soil quality, land use, groundwater level and salinity, irrigated infrastructure) were observed at local scale in Uzbekistan using moderate resolution imaging spectroradiometer (MODIS) time series data [17].

Despite the range of studies focused on vegetation changes in CA, no study has so far explicitly linked vegetation dynamics with several causative factors in an integrated manner at regional level to analyze inter-annual dynamics [18]. The reported studies focused mainly on trend analysis of satellite-based vegetation indices and correlated those on a pair-wise basis with likely drivers such as in the recent study by [19]. Analyses of pair-wise correlations between vegetation trends and their drivers, though useful, neither provide information on the impacts of driving factors on vegetation dynamics on an annual basis, nor do they consider the cumulative impact of several factors on inter-annual vegetation changes. Thus, the combined impact of different factors related to both environment and LULC on inter-annual vegetation dynamics should be examined in order to improve decision making on balancing environmental concerns (i.e., land degradation) with cropland intensification. In addition, the relative importance of the broad range of factors that contribute to the vegetation dynamics ongoing within CA in the context of simultaneous differences in land management practices and climatic parameters should be analyzed. This will enhance understanding of the relative contributions of the various driving factors responsible for vegetation change within similar geographical areas characterized by analogous management activities. To analyze the individual impact of factors on inter-annual vegetation dynamics while accounting for their interactions, we applied a statistical model, the generalized least squares (GLS) model [20], which is commonly used in econometrics but has been less frequently used in the field of remote sensing. 
Until now, only a limited number of studies have applied medium spatial resolution satellite data time series in the region. Such data sets are likely to be more applicable and accurate for monitoring fragmented CA drylands landscapes [11,21]. The spatial and temporal parameters of the MODIS sensor allow the characterization of vegetation dynamics at both a relatively high spatial detail $(250 \mathrm{~m}$ pixel size) and within seasonal to decadal time frames [22]. Finally, MODIS vegetation parameters and ground phenological reference data have been demonstrated to be highly correlated, increasing the utility of MODIS time series data for vegetation analyses [23].

In this study, we analyzed inter-annual vegetation dynamics at a regional level using a medium spatial resolution $(250 \mathrm{~m})$ MODIS-NDVI time-series data set. We integrated the remote sensing techniques with GLS modeling to obtain quantitative information on the contribution of mainly environmental factors to the vegetation dynamics observed across the CA region. This is the first region-based study to assess the complexity of various drivers in an integrated manner as determinants for vegetation change over longer time periods.

\section{Study Area and Datasets}

\subsection{Study Area}

The study area consists of five post-Soviet CA countries: Kazakhstan, Kyrgyzstan, Tajikistan, Turkmenistan, and Uzbekistan (Figure 1). The climate in this region is predominantly semi-arid and arid, and follows a distinct humidity gradient increasing south to north of Central Asia and has a sharply continental climate with high levels of variability. Mean winter temperatures throughout the region during last century have ranged between $-25^{\circ} \mathrm{C}$ and $+7^{\circ} \mathrm{C}$, while the mean summer temperatures were between $+2{ }^{\circ} \mathrm{C}$ and $+31^{\circ} \mathrm{C}$. In the mountain areas, the minimum temperatures can be as low as $-45^{\circ} \mathrm{C}$, and in the desert areas, the maximum temperatures can be as high as $+50{ }^{\circ} \mathrm{C}$ [24]. Similarly, the mean annual precipitation during the last century has ranged between $60 \mathrm{~mm}$ in the driest areas in southern Uzbekistan and northern Turkmenistan and $1180 \mathrm{~mm}$ in the mountainous areas of Kyrgyzstan and Tajikistan. The precipitation regime is characterized by uneven distribution and considerable rainfall variability over various time scales [25]. Maximum precipitation occurs during winter and early spring in most of the region and is meteorologically associated with the northward migration of the Iranian branch of the Polar front [26].

The study area includes three distinct sub-regional landscapes based on the Köppen climate classification as described by Kariyeva and van Leeuwen [27]: (1) northern semi-steppe and steppe sub-region; (2) mountainous sub-region and (3) central semi-desert/desert sub-region. Rainfed agriculture predominates in the northern and mountainous sub-regions, while large-scale irrigated agriculture is common in the central region. The most widespread crops in CA are cotton and winter wheat [17]. 


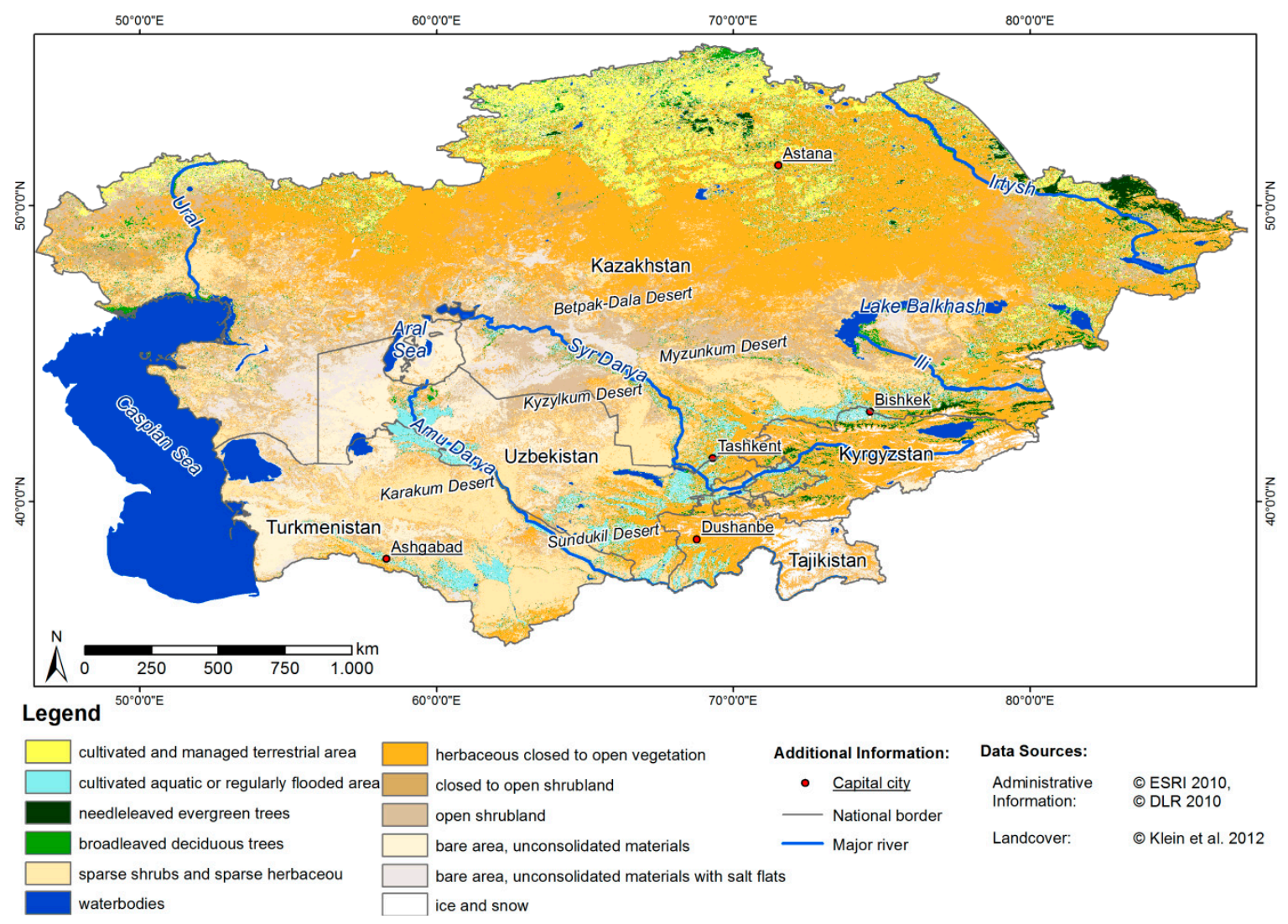

Figure 1. The study area: the post-Soviet Central Asia.

\subsection{Datasets}

The NDVI time-series data from the MODIS $250 \mathrm{~m}$ MOD13Q1 (Collection 5) product served as a primary input for this analysis. The 2000-2009 time period was chosen considering the availability of time series of the ancillary datasets. Especially, for our analysis, the availability of the accurate and reliable LULC maps was crucial, as those maps were used in GLS modeling for stratification by management type. Thus, we used LULC maps available for 2001 and 2009 by Klein et al. [28] which is currently the most accurate regional LULC product for CA and is also derived from MODIS data with spatial resolution of $250 \mathrm{~m}$.

The MOD13Q1 images are atmospherically corrected [25] and composed of optimal observations acquired during a 16-day period. Optimal observations are designated by evaluating overall pixel quality in terms of aerosol content, low view angle, and absence of clouds and/or cloud shadows [26]. In order to further reduce data noise levels resulting from cloud and cloud shadow contamination, the MODIS time-series datasets were smoothed with a Whittaker smoother algorithm [27].

A suite of ancillary data was also compiled and used principally to link quantitative vegetation cover dynamics with their causative factors. These data sets consist of climate data including mean monthly temperature, precipitation, snow cover dynamics, along with elevation data and LULC maps (Section 3.1).

\section{Methods}

\subsection{Data Compilation for Statistical Modelling}

The factors determining inter-annual vegetation dynamics in CA were identified based on a literature review and expert interviews. The principal factors for which data were available were 
utilized as inputs for the generalized least squares (GLS) random-effect (RE) models (Table 1). Our models were stratified based on both regional differences in climatic conditions and in management.

Table 1. Overview of the factors used for statistical modelling.

\begin{tabular}{cccc}
\hline Variable & Description & Time Step & Data Source \\
\hline L.ndvi_a0 & Lagged overall greenness & Yearly & MOD13Q1 \\
\hline temp_a0 & Overall temperature & Yearly & \\
temp_a1 & Peak temperature & Yearly & \\
temp_ph & Timing of peak temperature & Yearly & \\
L.temp_a0 & Lagged overall temperature & Yearly & \\
L.temp_a1 & Lagged peak temperature & Yearly & \\
L.temp_ph & Lagged timing of peak temperature & Yearly & CRU TS3.10 \\
prec_a0 & Overall precipitation & Yearly & \\
prec_a1 & Peak precipitation & Yearly & \\
prec_ph & Timing of peak precipitation & Yearly & \\
L.prec_a0 & Lagged overall precipitation & Yearly & \\
L.prec_a1 & Lagged peak precipitation & Yearly & \\
L.prec_ph & Lagged timing of peak precipitation & Yearly & \\
\hline snow & Stop of the snow cover season & Yearly & MOD10A1, \\
L.snow & Lagged stop of the snow cover season & Yearly & MYD10A1 \\
\hline elav & Slope & Time invariant & SRTM \\
\hline LU & Land use land cover & Year 2009 & MYD13Q1, \\
& & & MYD09Q1 \\
\hline
\end{tabular}

Consequently, we created separate models for three sub-regional landscapes: (1) northern semi-steppe and steppe sub-region; (2) mountainous sub-region and (3) central semi-desert/desert sub-region (Section 2.1). Management gradient was represented by the most widespread LULC classes in the region: non-irrigated cropland, irrigated cropland, and natural (unmanaged) vegetation (Section 3.3).

Corresponding factor maps were prepared for each factor (independent variables $x_{i}$ ). These maps were binary (factor presence $=1$; absence $=0$ ) and continuous; their spatial extent, $250 \mathrm{~m}$ cell size spatial resolution, map projection, and coordinate system were identical to the input MODIS data. The dependent variable $(y)$ represented the key vegetation metric of overall greenness or the mean annual NDVI. This metric is strongly correlated with seasonal vegetation production, and changes in overall greenness serve as effective general indicators for inter-annual vegetation productivity changes [29].

Vegetation activity is strongly linked to temperature and precipitation [3,4]. We used the best available temperature and precipitation data available for this region and for our observation period which is the updated high-resolution grids of monthly climatic observations (CRU TS3.10 Dataset) [30]. Monthly aggregated CRU observations are gridded at a resolution of $0.5^{\circ}$. The CRU TS3.10 Dataset is derived from archives of climate station records that have been subject to extensive manual and semi-automated quality control measures.

Snow dynamics are important for vegetation assessment, as they are associated with regional water availability. Snow cover refills the groundwater across the region and feeds glaciers in the mountainous parts of CA, which in turn are the main sources of water for the main rivers in the region such as the Amu Darya and Syr Darya rivers [31]. Among different seasonal parameters that describe snow dynamics, the timing of the end of the snow cover season (SCS) has the greatest influence upon subsequent freshwater availability [32]. This parameter defines flood events, droughts, and irrigation water availability in the downstream water network of CA, which subsequently impact vegetation activity. We used the SCS time series (2000-2009) collected from the Global SnowPack product [32]. 
Elevation is a predictor variable that directly influences vegetation distribution and growth. Though most of the topography of CA is levelled, mountains and highlands are present in the south of Tajikistan and Kyrgyzstan. To include the critical elevation parameter in our analysis we utilized a $90 \mathrm{~m}$ spatial resolution digital elevation model (DEM) acquired by the Shuttle Radar Topography Mission (SRTM). We derived slope values from the DEM, and these data were resampled to $250 \mathrm{~m}$ spatial resolution.

Land use is an important human-induced factor of vegetation dynamics. Information on regional land use and land cover (LULC) in CA was derived from 2001 and 2009 regional land cover maps [28]. These maps are based on the $250 \mathrm{~m}$ MODIS time series and include 12 LULC classes. We used the LULC map of 2001 to define management classes (i.e., irrigated, non-irrigated, and natural vegetation), and the map of 2009 was used as a categorical variable in the modelling process.

Previous research has documented a temporal lag between climate-related variables and vegetation activity in the CA region [14,33]. In order to quantify the impacts of these time-lagged variables on vegetation as well as to consider the mismatch between the climatic cycle (most precipitation falls outside of the vegetation growing season and within the winter months from December to February) and the nature of the products used (which are based on a calendar year), we have implemented a one-year lag variable for each of these factors.

\subsection{Calculating Seasonality Metrics from NDVI and Climatic Data}

To extract key seasonal metrics, harmonic regression was applied to the NDVI time series data, and the precipitation and the temperature data. Through a rearrangement of terms following the solution for a harmonic regression, the generalized seasonal curve can be formulated by [34]:

$$
y=a_{0}+a_{n} \sin \left(\frac{2 \pi n t}{T}+\varphi_{n}\right)
$$

where $\alpha_{n}$ are amplitudes and the $\varphi_{n}$ are phase angles ranging from $0^{\circ}$ to $360^{\circ}, \mathrm{n}$ is a harmonic.

Amplitude $0\left(a_{0}\right)$ is an overall metric, representing the mean annual value for NDVI/ temperature/precipitation. Amplitude $1\left(a_{1}\right)$ represents the magnitude or peak of annual temperature and precipitation. Phase $1\left(\varphi_{1}\right)$ refers to the timing of the annual peak defined by the position of the starting point of the corresponding sine wave of annual greenness. Phase image values potentially range from $0^{\circ}$ to $360^{\circ}$ such that each $30^{\circ}$ represents a shift of approximately one calendar month.

A well-fit model is not necessarily the same as a correct model. $R^{2}$ values can approach 0 under a correct model if the residual variance of $y$ is always close to the total variance of $y$ [35]. Numerous studies [36-38] identify harmonic regression as an appropriate model for our task, i.e., extraction of seasonal curve parameters and data smoothing. Thus, no additional verification of the model fit was required.

\subsection{Statistical Modelling}

To quantify the contribution of the factors to the overall inter-annual vegetation dynamics, we utilized generalized least-squares random effect (GLS-RE) model [17]. The GLS-RE model was selected as it is appropriate for the analysis of the panel data in which the behavior of entities, i.e., overall greenness, is observed through time. The rationale behind RE model is that the variation across entities is assumed to be random and uncorrelated with the independent variables included in the model. A random-effect GLS has the additional advantage of processing both temporally dynamic (e.g., temperature) and temporally static variables (e.g., elevation), as well as differences across the independent variables (i.e., factors), which may influence the dependent variable (i.e., overall greenness). The models were based on standardized data for cross-comparison of the factors and their impacts. To correct for serial correlation of lagged time-dependent variables, we used hierarchical model structure as implemented in Data Analysis and Statistical Software (STATA). 
The equation for the GLS-RE effects model is as follows:

$$
Y_{i t}=B_{i} X_{i t}+a_{i}+u_{i t}+e_{i t}
$$

$Y_{i t}$ is the dependent variable where $i=$ entity and $t=$ time,

$X_{i t}$ represents one independent variable,

$B_{i}$ is the coefficient for that $X$,

$a_{i}(i=1 \ldots . \mathrm{n})$ is the unknown intercept for each entity ( $n$ entity-specific intercepts),

$u_{i t}$ is the between-entity error,

$e_{i t}$ is the within-entity error.

To measure size effects and impact of the independent variables, we calculated the partial eta-squared $\left(\eta^{2}\right)$ estimates [39]. The $\eta^{2}$ estimate can be interpreted as the proportion of variance in the dependent variable that is attributable to each effect; and it is analogous to $R^{2}$ from multiple linear regressions.

We used the graphical representation to communicate the modeling results. The plots allow the enhanced, compact, and clear representation of the regression models' results in contrast to the traditionally used tables as well as they are able to display several regression models in a parallel fashion. Regression tables are meant to communicate two essential quantities: point estimates (coefficients) and uncertainty estimates (usually in the form of confidence intervals, standard errors, or null hypothesis tests). The coefplots (i.e., the graphs of point estimates and confidence intervals) were used in this study to show the same information as standard regression tables while adding the benefit of easy comparison of coefficients from one model and across the models and emphasizing effect size. A confidence interval effectively presents the same information as a null hypothesis significance test; for example, a 95\% confidence interval that does not include the null hypothesis (typically zero) is equivalent to a coefficient being statistically significant at $p<0.05$ [40]. Inclusion of the confidence intervals in the tabular presentation of the model's results is possible but such representation is usually difficult to read and interpret.

We have implemented a separate model for each land management type (i.e., irrigated cropland, non-irrigated cropland, and natural vegetation) within the northern, central, and mountain sub-regions of CA. A number of classes included in the source LULC maps were aggregated and/or referenced to represent the management gradient in this analysis. These classes were extracted from the LULC map of 2001 after consulting the map's authors. The LULC mapped class "Cultivated Aquatic or Regularly Flooded Area" was referenced for the modelling procedure as irrigated cropland; the mapped LULC class "Cultivated and Managed Terrestrial Area" was referenced as rainfed cropland. The modelled natural vegetation class is an aggregation of six LULC map classes: "Needleleaved Evergreen Trees", "Broadleaved Deciduous Trees", "Sparse Shrubs and Sparse Herbaceous", "Herbaceous Closed to Open Vegetation", "Closed to Open Shrubland", and "Open Shrubland" (Figure 1). The broad class natural vegetation incorporates classes describing forest, shrubland, and grassland vegetation. Shrubland and grassland are spectrally similar classes in CA and have similar responses to climatic and anthropogenic factors, while tree cover in the region is negligible as it does not exceed $4 \%$ of the study area [28].

\section{Results and Discussion}

\subsection{Distribution of Overall Greenness in Central Asia during 2000-2009}

Figure 2 shows the distribution of overall greenness over the CA region. The vegetation distribution in CA follows a distinct gradient. The higher overall greenness values were found in the north and southeast parts of the region. The lowest overall greenness values were located in the south (Figure $2 \mathrm{~b}, \mathrm{~d}$ ). This pattern reflects the distinct regional climatic gradient. 

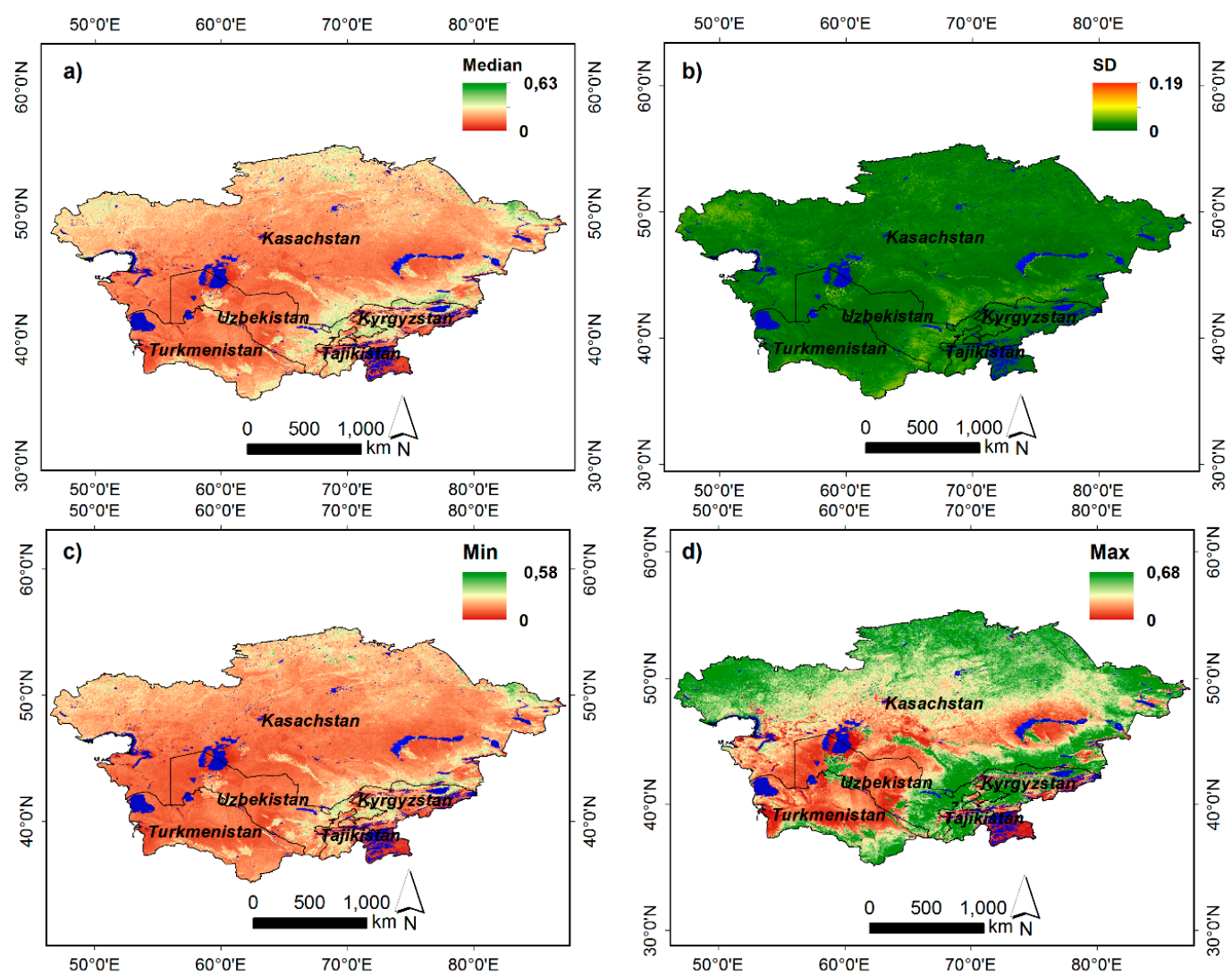

Figure 2. Spatially explicit descriptive statistics of overall greenness across post-Soviet Central Asia. These values were calculated from the 16-day $250 \mathrm{~m}$ Moderate Resolution Imaging Spectroradiometer (MODIS) Normalized Difference Vegetation Index (NDVI) time series for 2000-2009: (a) Median; (b) Standard deviation; (c) Minimum; (d) Maximum.

The northern semi-steppe and steppe sub-region has an area of almost $27 \times 10^{6} \mathrm{~km}^{2}$ and covers most of Kazakhstan. Vegetation dynamics in this sub-region are driven primarily by climatic regimes that follow a latitudinal gradient [40]. Rainfed croplands cover a $2 \times 10^{5} \mathrm{~km}^{2}$ area within CA. Due to favorable precipitation conditions of $200-400 \mathrm{~mm}$ annually almost all of the rainfed croplands of CA are located within the northern zone of the region [24]. In this part of CA, the Virgin Lands Campaign (1954-1961) converted the most suitable for agriculture rangelands into the cultivated cropland. After 1961, cultivated cropland was gradually expanded further southward to the lands characterized by lower agricultural suitability. Agricultural expansion to these marginal areas has resulted in land degradation and subsequent cropland abandonment which is still ongoing process in the region. The same processes have occurred within the irrigated cropland areas of the central and southern parts of $\mathrm{CA}$, forming green oases in the deserted areas shown with higher overall greenness values in Figure 2a,c [17,18].

Higher values of overall greenness were identified within the north and southeast parts of CA. These areas include the mountainous sub-region portions of Tajikistan and Kyrgyzstan. Vegetation growth patterns in this part of CA are constrained mainly by temperature thresholds and again follow a distinct latitudinal gradient. This part of CA is thought to be most severely impacted by the gradually increasing temperatures associated with global climate change. This temperature increase causes glacial melting, and the runoff feeds the region's principal rivers: the Amu Darya and Syr Darya [41]. The lowest overall greenness values were found in central and southern Turkmenistan and Uzbekistan. These areas lie within the central desert sub-region, which is the most arid part of the CA region. Total annual precipitation within this sub-region is as little as $100 \mathrm{~mm}$ (Figures 1 and 2). This area also includes the most extensive large-scale irrigated croplands in CA [42]. 


\subsection{Factors of Inter-Annual Vegetation Dynamics}

In order to compare the inter-annual vegetation dynamics factors by management type and regional landscape, nine GLS-RE models were analyzed based on standardized input data used to represent these factors (Figure 3). All models included 16 independent variables (see Table 1 ). $R^{2}$ values for all models tested were between 0.76 (northern non-irrigated zone) and 0.88 (central irrigated zone). These values suggest an overall good fit for the models.
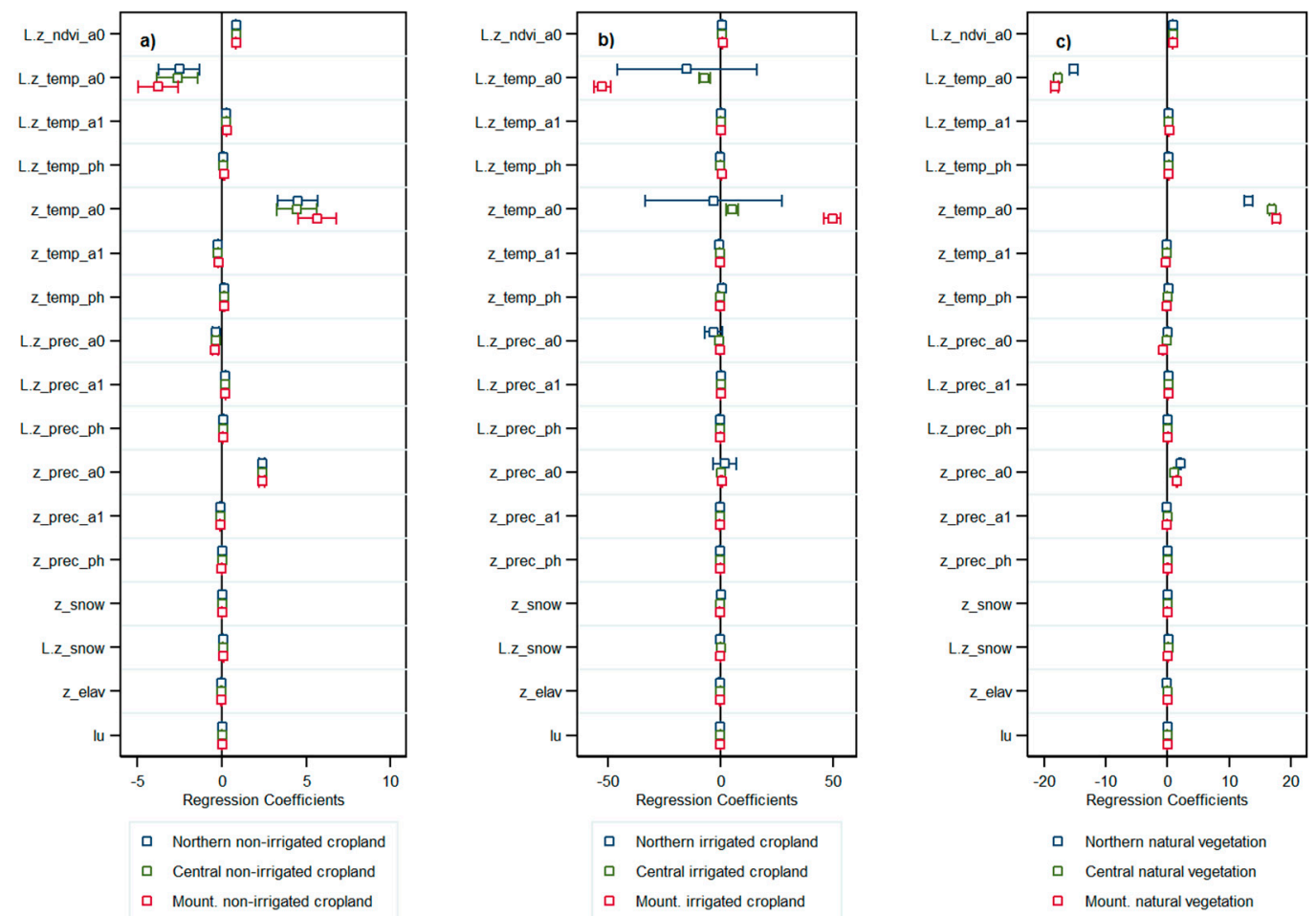

Figure 3. Coefplots of the generalized least-squares random effect models (GLS-RE) models tested to examine relationships between vegetation dynamics and standardized (denoted with " $z$ ") environmental factors in the study area: (a) Non-irrigated cropland; (b) Irrigated cropland; and (c) Natural vegetation. The squares represent the variable estimates and the horizontal lines depict 95\% confidence intervals. The range of parameter estimates is displayed on the $x$-axis, and each of the variable labels (Table 1) are displayed on the y-axis. For clarity, a vertical line has been drawn at the zero value.

\subsubsection{Environmental Factors of Inter-Annual Dynamics of Natural Vegetation and Rain Fed Croplands}

Overall, factors driving inter-annual vegetation dynamics were similar for the models of natural and within croplands non-irrigated vegetation. The factors related to temperature and precipitation had the greatest impact on inter-annual dynamics over different climatic sub-regions outside of irrigated croplands (Figure 3a,c). This result confirms the vast control of climatic variability over vegetation dynamics in CA. The highest impact of these variables could be explained by the harsh climatic conditions that are dominant across the region. These two level out the impact of the rest of the analyzed factors. This finding is also in line with the previous research that reported precipitation and temperature as the fundamental driving factors of vegetation growth within non-irrigated areas in CA $[8,40]$.

The $R^{2}$ coefficient for the north, central, and mountainous non-irrigated zone was calculated at 0.77 . This indicates an above average fit of the models for this zone. Factors of lagged mean temperature, mean temperature, and mean precipitation had the greatest relative impacts on vegetation for all 
non-irrigated cropland in the region. Similar results were observed for models of natural vegetation across other climatic sub-regions (Figure 3c). However, the impact of the mean precipitation factor was not as pronounced in other sub-regions as in non-irrigated croplands located in the northern sub-region of CA. This result is in the line with the study of Gessner et al. [14] who observed an acute sensitivity of vegetation to precipitation anomalies in areas receiving 100-400 $\mathrm{mm}$ of rainfall annually, as does the northern sub-region of CA.

For the natural vegetation and non-irrigated cropland classes across all climatic sub-regions (Table 2), the partial eta-squared $\left(\eta^{2}\right)$ values, which measure size effects and impact of the analyzed factors, show that the lagged overall greenness variable had the greatest impact on vegetation dynamics. This variable explained a higher proportion of the variance than any other factor: $72 \%$ for the northern non-irrigated zone and $83 \%$ for natural vegetation in the mountainous sub-region. This may be best explained by considering the function of vegetation as an integrated indicator of vegetation response to both environmental factors and land transformations related to the LULC changes [43,44]. In this manner, the overall greenness factor determined for the year prior to the year under analysis becomes particularly important. This single factor integrates multiple effects of several climatic and human induced factors which influenced ongoing inter-annual vegetation dynamics and were not explicitly included in the model variables.

Table 2. Variable effect magnitude expressed as partial eta-squared $\left(\eta^{2}\right)$ values for the calculated models.

\begin{tabular}{c|ccc|ccc|ccc}
\hline & \multicolumn{3}{|c|}{ Irrigated } & \multicolumn{3}{c|}{ Non-irrigated } & \multicolumn{3}{c}{ Natural Vegetation } \\
\hline Variable & N. & C. & M. & N. & C. & M. & N. & C. & M. \\
\hline L.ndvi_a0 & $\mathbf{2 9}$ & $\mathbf{8 7}$ & $\mathbf{8 4}$ & $\mathbf{7 2}$ & $\mathbf{7 2}$ & $\mathbf{7 3}$ & $\mathbf{7 4}$ & $\mathbf{7 8}$ & $\mathbf{8 3}$ \\
L.temp_a0 & 2 & 0 & $\mathbf{1 0}$ & 0 & 0 & 0 & 2 & $\mathbf{4}$ & $\mathbf{3}$ \\
L.temp_a1 & $\mathbf{8}$ & 1 & 2 & $\mathbf{5}$ & $\mathbf{5}$ & $\mathbf{6}$ & $\mathbf{4}$ & 2 & $\mathbf{7}$ \\
L.temp_ph & 2 & 0 & $\mathbf{7}$ & 0 & 0 & 0 & 1 & 2 & 1 \\
temp_a0 & 0 & 0 & $\mathbf{9}$ & 0 & 0 & 0 & $\mathbf{2}$ & $\mathbf{3}$ & $\mathbf{3}$ \\
temp_a1 & $\mathbf{8}$ & $\mathbf{2}$ & 3 & 3 & 3 & 3 & 2 & 2 & $\mathbf{6}$ \\
temp_ph & $\mathbf{8}$ & 1 & 0 & 0 & 0 & 0 & 0 & 0 & 1 \\
L.prec_a0 & 5 & 0 & 0 & 0 & 0 & 0 & 0 & 0 & 0 \\
L.prec_a1 & $\mathbf{1 8}$ & $\mathbf{3}$ & $\mathbf{6}$ & $\mathbf{6}$ & $\mathbf{6}$ & $\mathbf{6}$ & $\mathbf{4}$ & $\mathbf{5}$ & $\mathbf{4}$ \\
L.prec_ph & $\mathbf{1 0}$ & 0 & 0 & 1 & 1 & 0 & 1 & 0 & 0 \\
prec_a0 & 2 & 0 & 0 & $\mathbf{2}$ & $\mathbf{2}$ & $\mathbf{2}$ & $\mathbf{2}$ & 1 & 1 \\
prec_a1 & 1 & 1 & 3 & 1 & 1 & 1 & 1 & 1 & 2 \\
prec_ph & $\mathbf{6}$ & 0 & 0 & 0 & 0 & 0 & 0 & 0 & 0 \\
snow & 1 & 1 & 1 & 0 & 0 & 0 & 0 & 0 & 0 \\
L.snow & 5 & 1 & 0 & 0 & 0 & 0 & 1 & 1 & 0 \\
LULC & 0 & 0 & $\mathbf{4}$ & 0 & 1 & 1 & 0 & 0 & 0 \\
elav & $\mathbf{4}$ & 0 & 0 & 0 & 0 & 0 & 1 & 0 & 0 \\
\hline
\end{tabular}

* N. = northern, C. = central, M. = mountainous sub-regions. Orange color highlights the highest values of $\eta^{2}$ for every factor analyzed.

For northern and central non-irrigated zones, the other important factors as measured by partial eta-squared were the peak precipitation and lagged peak temperature (Table 2). For the mountainous sub-region, the peak temperature variable had significant effect on natural vegetation $\left(\eta^{2}=3\right)$, non-irrigated cropland $\left(\eta^{2}=3\right)$ and irrigated cropland $\left(\eta^{2}=6\right)$ compared to the other variables. In the mountainous sub-region vegetation patterns are distributed along altitude gradients and temperature thresholds are the main constraint on vegetation growth [27]. Overall fit of the models was high $\left(R^{2}=0.81\right)$ for the natural vegetation class and equal to or only marginally below values calculated for the northern sub-region $\left(R^{2}=0.81\right)$, the central sub-region and $\left(R^{2}=0.87\right)$ and the mountainous sub-region $\left(R^{2}=0.85\right)$.

\subsubsection{Factors of Inter-Annual Dynamics of Irrigated Croplands}

In contrast to non-irrigated vegetation in CA, within areas of irrigated croplands temperature had the highest relative impact as water availability is not typically a constraining factor for vegetation 
growth in these areas (Figure 3b) [41,45]. Even though most other factors including elevation, LULC, and end of SCS were also significant for all models related to irrigated agricultural area-with the exception of the model for northern irrigated zone-their impact on inter-annual vegetation dynamics was insignificant considering their small regression coefficients (Figures $3 \mathrm{~b}$ and 4; Table 2). For the mountainous sub-region, all variables related to lagged temperature and lagged peak precipitation were found to affect vegetation dynamics in contrast to the central and northern sub-regions. The LULC factor also had a significant impact, likely due to the fragmented landscapes and heterogeneous land use patterns typical for mountainous areas [46]. For the central sub-region, peak temperature was also an important factor along with lagged peak precipitation.

Among all models, the model for the northern irrigated zone demonstrated the greatest variability of the factors that impact vegetation dynamics (Figure 3b; Table 2). Results of this model document the impact of several variables: lagged SCS, elevation, and precipitation timing (both lagged and non-lagged). The large confidence intervals that cross the zero-line for the majority of the analyzed variables (Figure 4$)$ in the northern irrigated cropland areas suggest that the sample size $(n=54)$ for northern irrigated cropland should be increased for a more precise coefficient estimation. These results can also be an artifact of land use patterns present within the region. Irrigated areas in the northern part of the region typically take the form of relatively small, fragmented parcels extending over the entire sub-region [47]. Overall fit was good for irrigated cropland models: 0.80 for the northern sub-region, 0.88 for the central sub-region, and 0.87 for the mountainous sub-region.
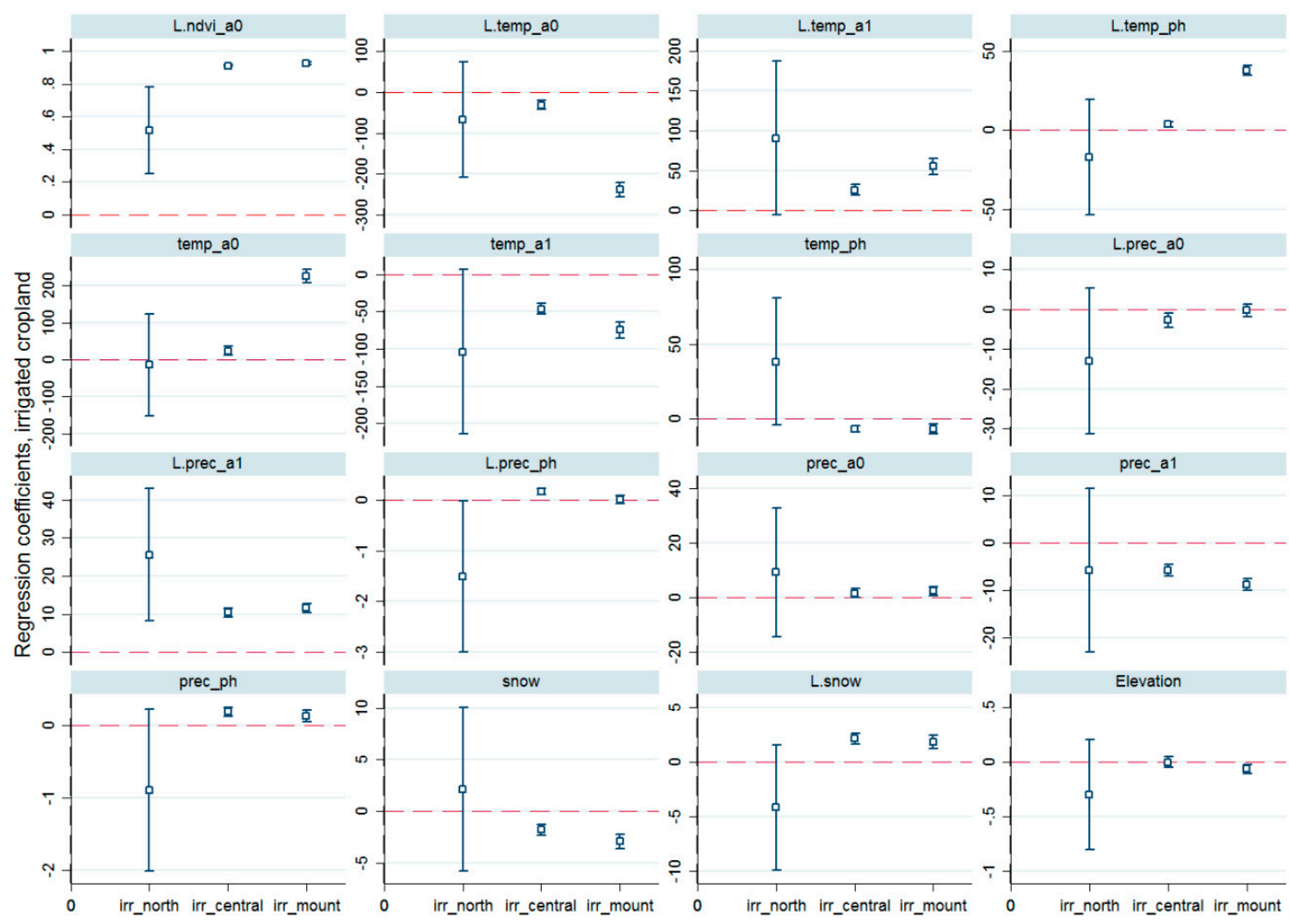

Figure 4. Regression coefficients of generalized least-squares random effect (GLS-RE) models depict relation of inter-annual vegetation dynamics and non-standardized factors within irrigated croplands of post-Soviet Central Asia. The squares represent the variable estimates and the vertical lines depict $95 \%$ confidence intervals. The range of parameter estimates is displayed on the y-axis, each of the variable labels (see Table 1) are displayed on the top of each individual plot, and names of different sub-regions are displayed on the x-axis. For clarity, a horizontal red line has been drawn at the zero value. ${ }^{*}$ irr_north $=$ northern irrigated, irr_central = central irrigated, irr_mount. = mountainous irrigated sub-regions. 
The fundamental findings of this study support previous region-wide research $[12,14,19]$. Unlike the previous studies, we applied a novel integrated methodology to measure and compare the impact of different environmental factors on vegetation dynamics rather than by the more traditional approach using pair-wise correlations between vegetation and possible drivers used in the cited papers. We also separated the impacts of environmental factors across climatic and management gradients which provide a better insight on differences and similarities of vegetation drivers considering the large area of the study region.

\subsubsection{Other Factors of Inter-Annual Vegetation Dynamics of Central Asia}

This study could have benefitted from the integration of additional causal factors related to regional socio-economic conditions and management characteristics such as demographic dynamics, income distribution, and agricultural land use (grazing intensity, cropping intensity, cropping patterns, and distribution of abandoned cropland). The processes of cropland abandonment due to the dissolution of the Soviet Union and the transition to a market economy are usually named as the underlying causes of the sharp decline in agricultural land use in the 1990s across CA [42]. The disintegration of value chain supplies, loss of previously guaranteed markets, and failing price relationships between inputs and outputs during the transition period triggered the decline in agricultural production and the cropland abandonment. Even though the land abandonment is frequently reported in CA [48,49], there is no reliable data on the extent and distribution of the abandoned land across the region. This situation is further aggravated by increasing frequency of droughts in the region and ongoing land degradation. The process of land degradation in CA is caused by numerous proximate and underlying drivers. The unsustainable agricultural practices, inadequate maintenance of irrigation and drainage networks, and overgrazing cause land degradation and abandonment which, in turn, impact vegetation dynamics in the region [24,42]. The trend analysis of MODIS-NDVI (250 m) time series by [43] for years 2000-2011 illustrates a prevailing negative trends within northern and north-western part of Kazakhstan as well as within the main irrigated areas of Uzbekistan and Turkmenistan where most of the abandoned and degraded lands are reportedly located [17].

At the same time, the mentioned land-related processes are triggered by current and former policies, institutional arrangements and overall socio-economic situation in the region. The former Soviet policies of cotton and grain self-sufficiency had led to a massive expansion of irrigated cotton and rainfed wheat production to marginal, often not suitable for cropping, areas. Consequently, there is a lack of resources and incentives to maintain irrigation and drainage networks and manage the expanded croplands in these marginal areas under the conditions of market economy [24]. The farm reform in some CA countries, such as Uzbekistan, has led to a mismatch between new farm sizes with the irrigation networks in place, which were planned for large-scale centralized farming. This has resulted in an institutional vacuum on sharing the responsibilities for the maintenance of the irrigation and drainage systems [50]. Other key underlying drivers of vegetation dynamics in the region include land tenure insecurity, breakdown of institutions regulating an access to rangeland resources and population growth. The incorporation of the full range of the casual factors in the quantitative analysis of vegetation dynamics described in this paper is, however, currently constrained by availability of corresponding datasets for multiple years as well as by current limited access for general public to existing socio-economic information in the region.

\section{Conclusions}

In this paper, we presented an analysis of inter-annual vegetation dynamics over post-Soviet Central Asia using a ten year (2000-2009) time-series of medium spatial resolution $(250 \mathrm{~m})$ MODIS-NDVI data. The key vegetation parameter of overall greenness was statistically linked to its potential environmental drivers in order to quantify information on the contribution of various factors relevant to the observed inter-annual vegetation dynamics. This analysis was conducted using 
partially-explicit regression models across various land management regimes: irrigated croplands, non-irrigated croplands, and naturally vegetated areas. The analysis was also performed within the context of climatic gradients represented by three principal regional landscapes (termed sub-regions): northern, central, and mountainous.

We have concluded the factors which drive vegetation dynamics differs only slightly across climatic and management gradients in the region. Non-irrigated croplands and naturally vegetated areas within northern and central sub-regions were impacted largely by variables related to both temperature and precipitation. Variables related to temperature had the greatest effects in irrigated areas across all sub-regions and for all types of vegetation cover within mountainous landscapes. The remaining causal factors were determined to be relatively insignificant. In addition, vegetation dynamics in CA are caused by numerous proximate and underlying drivers related to socio-economic and political situation in the countries of the region. The unsustainable agricultural practices, inadequate maintenance of irrigation and drainage networks, overgrazing cause land degradation and widespread land abandonment which have an impact on vegetation dynamics in CA. At the same time, the mentioned land-related processes are triggered by current and former policies, institutional arrangements and overall socio-economic situation in the region.

Finally, we conclude that $250 \mathrm{~m}$ MODIS data, when appropriately processed and aggregated in a time series and utilized in combination with appropriate and accurate ancillary datasets can serve as a suitable basis for development of information regarding inter-annual vegetation dynamics. Such information can be most valuable for the assessments of vegetation status across environmental and management gradients. It can also be utilized to identify and characterize some of the impacts on vegetation that are consequences of climate variability and land uses.

The integrated approach applied in this study, based on regression modelling and satellite data time series analysis enabled an inclusive evaluation of vegetation dynamics at the regional level. The models made it possible to address the various causal factors on an individual basis, and to quantitatively describe their impacts on inter-annual vegetation dynamics. This approach can be further improved by adding to the models socio-economic and institution drivers.

Acknowledgments: We would like to thank to Guido Lüchters for his advice on the statistical analysis, to Igor Klein for the provision of the regional land use and land cover maps, to Francesko Vuolo for MODIS data processing. We are very grateful to Joe Scepan for his help on the paper preparation.

Author Contributions: Olena Dubovyk designed paper, conducted analysis and wrote the paper. Andreas Dietz contributed to analysis. All authors contributed to the concept development, related discussions and paper writing.

Conflicts of Interest: The authors declare no conflict of interest.

\section{References}

1. Mueller, L.; Suleimenov, M.; Karimov, A.; Qadir, M.; Saparov, A.; Balgabayev, N.; Helming, K.; Lischeid, G. Land and water resources of Central Asia, their utilisation and ecological status. In Novel Measurement and Assessment Tools for Monitoring and Management of Land and Water Resources in Agricultural Landscapes of Central Asia; Mueller, L., Saparov, A., Lischeid, G., Eds.; Springer International Publishing: Cham, Switzerland, 2014; pp. 3-59.

2. Karthe, D.; Chalov, S.; Borchardt, D. Water resources and their management in Central Asia in the early twenty first century: Status, challenges and future prospects. Environ. Earth Sci. 2014, 73, 487-499. [CrossRef]

3. Löw, F.; Fliemann, E.; Abdullaev, I.; Conrad, C.; Lamers, J.P.A. Mapping abandoned agricultural land in Kyzylorda, Kazakhstan using satellite remote sensing. Appl. Geogr. 2015, 62, 377-390. [CrossRef]

4. Tüshaus, J.; Dubovyk, O.; Khamzina, A.; Menz, G. Comparison of medium spatial resolution ENVISAT-MERIS and Terra-MODIS time series for vegetation decline analysis: A case study in Central Asia. Remote Sens. 2014, 6, 5238-5256. [CrossRef]

5. Siegfried, T.; Bernauer, T.; Guiennet, R.; Sellars, S.; Robertson, A.; Mankin, J.; Bauer-Gottwein, P.; Yakovlev, A. Will climate change exacerbate water stress in Central Asia? Clim. Chang. 2012, 112, 881-899. [CrossRef] 
6. Fensholt, R.; Proud, S.R. Evaluation of earth observation based global long term vegetation trends-Comparing GIMMS and MODIS global NDVI time series. Remote Sens. Environ. 2012, 119, 131-147. [CrossRef]

7. Landmann, T.; Dubovyk, O. Spatial analysis of human-induced vegetation productivity decline over Eastern Africa using a decade (2001-2011) of medium resolution MODIS time-series data. Int. J. Appl. Earth Obs. Geoinf. 2014, 33, 76-82. [CrossRef]

8. Klein, I.; Gessner, U.; Künzer, C. Generation of up to date land cover maps for Central Asia. In Novel Measurement and Assessment Tools for Monitoring and Management of Land and Water Resources in Agricultural Landscapes of Central Asia; Mueller, L., Saparov, A., Lischeid, G., Eds.; Springer International Publishing: Cham, Switzerland, 2014; pp. 329-346.

9. Bajocco, S.; Angelis, A.; Perini, L.; Ferrara, A.; Salvati, L. The impact of land use/land cover changes on land degradation dynamics: A Mediterranean case study. Environ. Manag. 2012, 49, 980-989. [CrossRef] [PubMed]

10. Walker, J.J.; de Beurs, K.M.; Henebry, G.M. Land surface phenology along urban to rural gradients in the US Great Plains. Remote Sens. Environ. 2015, 165, 42-52. [CrossRef]

11. Dubovyk, O.; Landmann, T.; Erasmus, B.F.N.; Tewes, A.; Schellberg, J. Monitoring vegetation dynamics with medium resolution MODIS-EVI time series at sub-regional scale in Southern Africa. Int. J. Appl. Earth Obs. Geoinf. 2015, 38, 175-183. [CrossRef]

12. Zhou, Y.; Zhang, L.; Fensholt, R.; Wang, K.; Vitkovskaya, I.; Tian, F. Climate contributions to vegetation variations in Central Asian drylands: Pre- and post-USSR collapse. Remote Sens. 2015, 7, 2449-2470. [CrossRef]

13. Kariyeva, J.; van Leeuwen, W.J.D. Phenological dynamics of irrigated and natural drylands in Central Asia before and after the USSR collapse. Agric. Ecosyst. Environ. 2012, 162, 77-89. [CrossRef]

14. Gessner, U.; Naeimi, V.; Klein, I.; Kuenzer, C.; Klein, D.; Dech, S. The relationship between precipitation anomalies and satellite-derived vegetation activity in Central Asia. Glob. Planet. Chang. 2013, 110, 74-87. [CrossRef]

15. Propastin, P.A.; Kappas, M.; Muratova, N.R. Inter-annual changes in vegetation activities and their relationship to temperature and precipitation in Central Asia from 1982 to 2003. J. Environ. Inf. 2008, 12, 75-87. [CrossRef]

16. De Beurs, K.M.; Henebry, G.M. Land surface phenology, climatic variation, and institutional change: Analyzing agricultural land cover change in Kazakhstan. Remote Sens. Environ. 2004, 89, 497-509. [CrossRef]

17. Dubovyk, O. Multi-scale Targeting of Land Degradation in Northern Uzbekistan Using Satellite Remote Sensing. Ph.D. Thesis, University of Bonn, Bonn, Germany, 2013.

18. Dubovyk, O.; Menz, G.; Conrad, C.; Kan, E.; Machwitz, M.; Khamzina, A. Spatio-temporal analyses of cropland degradation in the irrigated lowlands of Uzbekistan using remote-sensing and logistic regression modeling. Environ. Monit. Assess. 2013, 185, 4775-4790. [CrossRef] [PubMed]

19. De Beurs, K.M.; Henebry, G.M.; Owsley, B.C.; Sokolik, I. Using multiple remote sensing perspectives to identify and attribute land surface dynamics in Central Asia 2001-2013. Remote Sens. Environ. 2015, 170, 48-61. [CrossRef]

20. Allison, P.D. Fixed Effects Regression Models; SAGE Publications: London, UK, 2009.

21. Walker, J.J.; de Beurs, K.M.; Wynne, R.H. Dryland vegetation phenology across an elevation gradient in Arizona, USA, investigated with fused MODIS and Landsat data. Remote Sens. Environ. 2014, 144, 85-97. [CrossRef]

22. Ganguly, S.; Friedl, M.A.; Tan, B.; Zhang, X.; Verma, M. Land surface phenology from MODIS: Characterization of the Collection 5 global land cover dynamics product. Remote Sen. Environ. 2010, 114, 1805-1816. [CrossRef]

23. Shuai, Y.; Schaaf, C.; Zhang, X.; Strahler, A.; Roy, D.; Morisette, J.; Wang, Z.; Nightingale, J.; Nickeson, J.; Richardson, A.; et al. Daily MODIS $500 \mathrm{~m}$ reflectance anisotropy direct broadcast (DB) products for monitoring vegetation phenology dynamics. Int. J. Remote Sens. 2013, 34, 5997-6016. [CrossRef]

24. Mirzabaev, A.; Goedecke, J.; Dubovyk, O.; Djanibekov, U.; Le, Q.; Aw-Hassan, A. Economics of land degradation in Central Asia. In Economics of Land Degradation and Improvement-A Global Assessment for Sustainable Development; Nkonya, E., Mirzabaev, A., von Braun, J., Eds.; Springer International Publishing: Cham, Switzerland, 2016; pp. 261-290. 
25. Xu, L.; Zhou, H.; Du, L.; Yao, H.; Wang, H. Precipitation trends and variability from 1950 to 2000 in arid lands of Central Asia. J. Arid Land 2015, 7, 514-526. [CrossRef]

26. Lioubimtseva, E.; Henebry, G.M. Climate and environmental change in arid Central Asia: Impacts, vulnerability, and adaptations. J. Arid Environ. 2009, 73, 963-977. [CrossRef]

27. Kariyeva, J.; van Leeuwen, W.J.D. Environmental drivers of NDVI-based vegetation phenology in Central Asia. Remote Sens. 2011, 3, 203-246. [CrossRef]

28. Klein, I.; Gessner, U.; Kuenzer, C. Regional land cover mapping and change detection in Central Asia using MODIS time-series. Appl. Geogr. 2012, 35, 219-234. [CrossRef]

29. Le, Q.B.; Tamene, L.; Vlek, P.L.G. Multi-pronged assessment of land degradation in West Africa to assess the importance of atmospheric fertilization in masking the processes involved. Glob. Planet. Chang. 2012, 92, 71-81. [CrossRef]

30. Harris, I.; Jones, P.D.; Osborn, T.J.; Lister, D.H. Updated high-resolution grids of monthly climatic observations-The CRU TS3.10 Dataset. Int. J. Climatol. 2013, 34, 623-642. [CrossRef]

31. Glantz, M.H. Water, climate, and development issues in the Amu Darya Basin. Mitig. Adapt. Strateg. Glob. Chang. 2005, 10, 23-50. [CrossRef]

32. Dietz, A.J.; Kuenzer, C.; Dech, S. Global snowpack: A new set of snow cover parameters for studying status and dynamics of the planetary snow cover extent. Remote Sens. Lett. 2015, 6, 844-853. [CrossRef]

33. Dietz, A.J.; Kuenzer, C.; Conrad, C. Snow-cover variability in Central Asia between 2000 and 2011 derived from improved MODIS daily snow-cover products. Int. J. Remote Sens. 2013, 34, 3879-3902. [CrossRef]

34. Eastman, R.; Sangermano, F.; Ghimire, B.; Zhu, H.; Chen, H.; Neeti, N.; Cai, Y.; Machado, E.A.; Crema, S.C. Seasonal trend analysis of image time series. Int. J. Remote Sens. 2009, 30, 2721-2726. [CrossRef]

35. Freedman, D. Statistical Models: Theory and Practice; Cambridge University Press: Cambridge, UK, 2006.

36. Bradley, B.A.; Jacob, R.W.; Hermance, J.F.; Mustard, J.F. A curve fitting procedure to derive inter-annual phenologies from time series of noisy satellite NDVI data. Remote Sens. Environ. 2007, 106, 137-145. [CrossRef]

37. Neeti, N.; Rogan, J.; Christman, Z.; Eastman, J.R.; Millones, M.; Schneider, L.; Nickl, E.; Schmook, B.; Turner, B.L.; Ghimire, B. Mapping seasonal trends in vegetation using AVHRR-NDVI time series in the Yucatãjn Peninsula, Mexico. Remote Sens. Lett. 2012, 3, 433-442. [CrossRef]

38. De Jong, R.; de Bruin, S.; de Wit, A.; Schaepman, M.E.; Dent, D.L. Analysis of monotonic greening and browning trends from global NDVI time-series. Remote Sens. Environ. 2011, 115, 692-702. [CrossRef]

39. Kerlinger, F.N. Foundations of Behavioral Research; Holt, Rinehart \& Winston: New York, NY, USA, 1964.

40. Kariyeva, J.; van Leeuwen, W.J.D.; Woodhouse, C.A. Impacts of climate gradients on the vegetation phenology of major land use types in Central Asia (1981-2008). Front. Earth Sci. 2012, 6, 206-225. [CrossRef]

41. Mirzabaev, A. Climate Volatility and Change in Central Asia: Economic Impacts and Adaptation. Ph.D. Thesis, University of Bonn, Bonn, Germany, 2013.

42. Aw-Hassan, A.; Korol, V.; Nishanov, N.; Djanibekov, U.; Dubovyk, O.; Mirzabaev, A. Economics of land degradation and improvement in Uzbekistan. In Economics of Land Degradation and Improvement-A Global Assessment for Sustainable Development; Nkonya, E., Mirzabaev, A., von Braun, J., Eds.; Springer International Publishing: Cham, Switzerland, 2016; pp. 651-682.

43. Dubovyk, O.; Landmann, T.; Erasmus, B.; Thonfeld, F.; Schellberg, J.; Menz, G. Observing vegetation dynamics at medium spatial scale: Lessons from Africa and Asia. In Proceedings of the 2015 IEEE International Geoscience and Remote Sensing Symposium (IGARSS), Milan, Italy, 26-31 July 2015; pp. 3969-3972.

44. Lu, L.; Guo, H.; Kuenzer, C.; Klein, I.; Zhang, L.; Li, X. Analyzing phenological changes with remote sensing data in Central Asia. IOP Conf. Ser.: Earth Environ. Sci. 2014, 17, 012005. [CrossRef]

45. Dukhovny, V.A.; de Schutter, J. Water in Central Asia: Past, Present, Future; CRC Press: London, UK, 2011.

46. Shigaeva, J.; Kollmair, M.; Niederer, P.; Maselli, D. Livelihoods in transition: Changing land use strategies and ecological implications in a post-Soviet setting (Kyrgyzstan). Cent. Asian Surv. 2007, 26, 389-406. [CrossRef]

47. Bucknall, J.; Klytchnikova, I.; Lampietti, J.; Lundell, M.; Scatasta, M.; Thurman, M. Irrigation in Central Asia: Social, Economic and Environmental Considerations; World Bank: Washington, DC, USA, 2003. 
48. Kraemer, R.; Prishchepov, A.V.; Müller, D.; Kuemmerle, T.; Radeloff, V.C.; Dara, A.; Terekhov, A.; Frühauf, M. Long-term agricultural land-cover change and potential for cropland expansion in the former Virgin Lands area of Kazakhstan. Environ. Res. Lett. 2015, 10, 054012. [CrossRef]

49. Meyfroidt, P.; Schierhorn, F.; Prishchepov, A.V.; Müller, D.; Kuemmerle, T. Drivers, constraints and trade-offs associated with recultivating abandoned cropland in Russia, Ukraine and Kazakhstan. Glob. Environ. Chang. 2016, 37, 1-15. [CrossRef]

50. Djanibekov, N.; Bobojonov, I.; Lamers, J.A. Farm reform in Uzbekistan. In Cotton, Water, Salts and Soums; Martius, C., Rudenko, I., Lamers, J.P.A., Vlek, P.L.G., Eds.; Springer Netherlands: Dordrecht, The Netherlands, 2012; pp. 95-112.

() 2016 by the authors; licensee MDPI, Basel, Switzerland. This article is an open access article distributed under the terms and conditions of the Creative Commons Attribution (CC-BY) license (http://creativecommons.org/licenses/by/4.0/). 